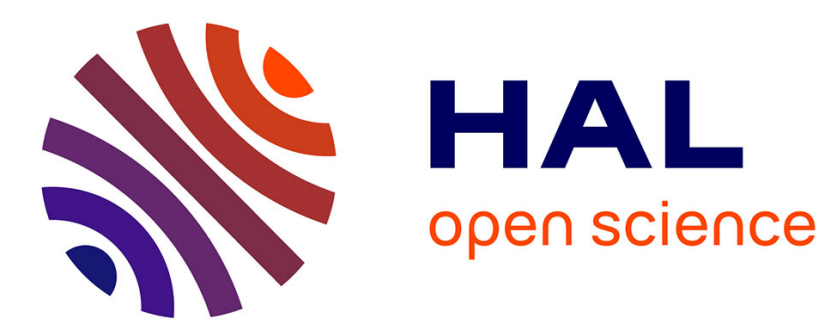

\title{
Thin cylindrical conductivity inclusions in a 3-dimensional domain: polarization tensor and unique determination from boundary data
}

Elena Beretta, Yves Capdeboscq, Elisa Francini

\section{- To cite this version:}

Elena Beretta, Yves Capdeboscq, Elisa Francini. Thin cylindrical conductivity inclusions in a 3dimensional domain: polarization tensor and unique determination from boundary data. Inverse Problems, 2009, 25, pp.065004. 10.1088/0266-5611/25/6/065004 . hal-00342730

\section{HAL Id: hal-00342730 \\ https://hal.science/hal-00342730}

Submitted on 30 Nov 2008

HAL is a multi-disciplinary open access archive for the deposit and dissemination of scientific research documents, whether they are published or not. The documents may come from teaching and research institutions in France or abroad, or from public or private research centers.
L'archive ouverte pluridisciplinaire HAL, est destinée au dépôt et à la diffusion de documents scientifiques de niveau recherche, publiés ou non, émanant des établissements d'enseignement et de recherche français ou étrangers, des laboratoires publics ou privés. 


\title{
THIN CYLINDRICAL CONDUCTIVITY INCLUSIONS IN A 3-DIMENSIONAL DOMAIN: POLARIZATION TENSOR AND UNIQUE DETERMINATION FROM BOUNDARY DATA
}

\author{
ELENA BERETTA , YVES CAPDEBOSCQ , AND ELISA FRANCINI
}

\begin{abstract}
We consider a 3-dimensional conductor containing an inclusion that can be represented as a cylinder with fixed axis and a small basis. As the size of the basis of the cylinder approaches zero, the voltage perturbation can be described by means of a polarization tensor. We give an explicit characterization of the polarization tensor of cylindrical inclusions in terms of the polarization tensor of its base, and we use this result to show that the axis of the inclusion can be uniquely determined by boundary values of the voltage perturbation.
\end{abstract}

\section{INTRODUCTION}

Let $\Omega$ be an open bounded smooth domain in $\mathbb{R}^{3}$ occupied by a conducting material, and let $\gamma_{0}: \Omega \rightarrow \mathbb{R}^{+}$represent the conductivity in $\Omega$.

If we assign a current $g$ on $\partial \Omega$ such that $\int_{\partial \Omega} g d \sigma=0$, the voltage potential generated by this current is the solution $u_{0}$ to

$$
\left\{\begin{aligned}
\operatorname{div}\left(\gamma_{0} \nabla u_{0}\right) & =0 \text { in } \Omega \\
\gamma_{0} \frac{\partial u_{0}}{\partial n} & =g \text { on } \partial \Omega \\
\int_{\partial \Omega} u_{0} d \sigma & =0
\end{aligned}\right.
$$

where last condition ensures the unique determination of the solution.

Let us suppose that $\Omega$ contains a small inclusion $\omega_{\epsilon}$, made of a different material with conductivity $\gamma_{1}: \Omega \rightarrow \mathbb{R}^{+}$. The perturbed conductivity is given by

$$
\gamma_{\epsilon}(x)= \begin{cases}\gamma_{0}(x) & x \in \Omega \backslash \omega_{\epsilon}, \\ \gamma_{1}(x) & x \in \omega_{\epsilon},\end{cases}
$$

If we apply the same current $g$ on the boundary of the body containing the inclusion, the resulting potential is the solution $u_{\epsilon}$ to the boundary value problem

$$
\left\{\begin{aligned}
\operatorname{div}\left(\gamma_{\epsilon} \nabla u_{\epsilon}\right) & =0 \text { in } \Omega, \\
\gamma_{\epsilon} \frac{\partial u_{\epsilon}}{\partial n} & =g \text { on } \partial \Omega, \\
\int_{\partial \Omega} u_{\epsilon} d \sigma & =0 .
\end{aligned}\right.
$$

In recent years, a considerable amount of work has been dedicated to the case of small inclusions, that is, to subsets $\omega_{\epsilon}$ whose Lebesgue measure tends to zero with $\epsilon$. When this happens, the perturbation of the voltage potential is very little, in the sense that $u_{\epsilon}$ converges to $u_{0}$ in the $H^{1}(\Omega)$ norm. A number of asymptotic formulas have been proved for the asymptotic expansion of $u_{\epsilon}-u_{\left.0\right|_{\partial \Omega}}$ with respect to $\epsilon$ for a variety of geometries. We recall here a general, geometry independent, result due to Capdeboscq \& Vogelius [7]. Assume that

$$
\left|\omega_{\epsilon}\right|^{-1} 1_{\omega_{\epsilon}}(\cdot) \text { converges in the sense of measure to } \mu \text { when }\left|\omega_{\epsilon}\right| \rightarrow 0 \text {. }
$$


Let $N$ denote the Neumann function of the unperturbed domain: given $y \in \Omega$, let $N(\cdot, y)$ be the solution to

$$
\left\{\begin{aligned}
\operatorname{div}_{x}\left(\gamma_{0}(x) \nabla_{x} N(x, y)\right) & =\delta_{y}(x) \text { for } x \in \Omega \\
\gamma_{0}(x) \frac{\partial N}{\partial n_{x}}(x, y) & =\frac{1}{|\partial \Omega|} \text { for } x \in \partial \Omega \\
\int_{\partial \Omega} N(x, y) d \sigma_{x} & =0
\end{aligned}\right.
$$

This function may be extended by continuity to $\partial \Omega$ and may also be defined as the solution to

$$
\left\{\begin{aligned}
\operatorname{div}_{x}\left(\gamma_{0}(x) \nabla_{x} N(x, y)\right) & =0 \text { for } x \in \Omega \\
\gamma_{0}(x) \frac{\partial N}{\partial n_{x}}(x, y) & =-\delta_{y}+\frac{1}{|\partial \Omega|} \text { for } x \in \partial \Omega \\
\int_{\partial \Omega} N(x, y) d \sigma_{x} & =0 .
\end{aligned}\right.
$$

The main result in [7] is the following:

Theorem 1.1. Assume (4) holds. There exists a tensor $\left\{M_{i j}\right\}_{i, j=1}^{3} \in L^{2}(\Omega, d \mu)$ such that, for $g \in H^{-1 / 2}(\partial \Omega)$ satisfying $\int_{\partial \Omega} g d \sigma=0$, if we denote by $u_{\epsilon}$ and $u_{0}$ the solutions to boundary value problems (1) and (3) respectively, we have that, for $y \in \partial \Omega$,

$$
\left(u_{\epsilon}-u_{0}\right)(y)=\left|\omega_{\epsilon}\right| \sum_{i, j=1}^{3} \int_{\Omega}\left(\gamma_{1}-\gamma_{0}\right)(x) M_{i j}(x) \frac{\partial u_{0}}{\partial x_{i}}(x) \frac{\partial N}{\partial x_{j}}(x, y) d \mu(x)+o\left(\left|\omega_{\epsilon}\right|\right) .
$$

The $o\left(\left|\omega_{\epsilon}\right|\right)$ term is such that $\left|\omega_{\epsilon}\right|^{-1}\left\|o\left(\left|\omega_{\epsilon}\right|\right)\right\|_{L^{\infty}(\partial \Omega)}$ converges to zero as $\epsilon$ tends to zero, uniformly on $\left\{g \in H^{-1 / 2}(\partial \Omega): \int_{\partial \Omega} g d \sigma=0,\|g\|_{L^{2}(\partial \Omega)} \leq 1\right\}$.

The symmetric tensor $M$ is the signature of the inclusion and it is called the polarization tensor. Later on, we will give more insight of how this tensor can be constructed. The concept of polarization tensor appears in various contexts. The term was coined by Polya, Schiffer \& Szegö $[18,17]$. Polarization tensors are well known in the theory of homogenization as the low volume fraction limit of the effective properties of the dilute two phase composites $[13,14,16]$ (see [14] for an extensive list of references).

Explicit formulas for the polarization tensor are available in the case of diametrically small inclusions, i.e. those inclusions that can be written as $\omega_{\epsilon}=z+\epsilon B$ where $z$ is a point in $\Omega$ and $B$ is a bounded domain centered at the origin (see [3]). Even in the case of "thin" inclusion, that can be described as small neighborhood of hypersurfaces (a curve in $\mathbb{R}^{2}$ or a surface in $\mathbb{R}^{3}$ ), the polarization tensor has been explicitly characterized (see [5]).

In this work, we want to consider inclusions that can be represented as small neighborhood of a line segment in a 3-dimensional domain. For these cylindrically shaped inclusions we give an explicit description of the polarization tensor. This model has many possible applications, for example in non-destructive testing of material and in geophysical prospection. We also want to look at this problem with the point of view of inverse problems. This approach was initiated by Friedman \& Vogelius [10] who first used the polarization tensor for the detection of small inclusions. After that there have been many significant developments in this direction. For more information on this subject we refer to recent books [3, 4] and references therein.

In Section 2 we will set up general assumptions and recall the definition of polarization tensor. In Section 3 we will state and prove our main result for cylindrical inclusions. In Section 4 we observe that the asymptotic formula that we derive in Section 3 is useful for the reconstruction of the inclusion from boundary data. In particular we show that boundary data of the second order term of the expansion uniquely determine the axis of the cylinder. 


\section{General assumptions}

In all that follows we will assume that our inclusions $\omega_{\epsilon}$ are contained in a compact set $K_{0} \subset \Omega$, with positive distance from $\partial \Omega$. The Borel measure $\mu$ defined by (4) will then be concentrated on $K_{0}$.

We will assume that both $\gamma_{0}$ and $\gamma_{1}$ are smooth functions in $\Omega$ and that, for some positive constant $c_{0}$, we have

$$
c_{0}<\gamma_{i}(x)<\frac{1}{c_{0}}, \quad \text { for } \quad x \in \Omega, \quad i=0,1 .
$$

The polarization tensor $M$ can be defined in several ways. In [7] it is defined by means of the following auxiliary problem.

For $j=1,2,3$, let $e_{j}$ denote the coordinate directions and let $v_{\epsilon}^{j} \in H^{1}(\Omega)$ be defined by

$$
\left\{\begin{aligned}
\operatorname{div}\left(\gamma_{\epsilon} \nabla v_{\epsilon}^{j}\right) & =\operatorname{div}\left(\gamma_{0} e_{j}\right) \text { in } \Omega, \\
\gamma_{\epsilon} \frac{\partial v_{\epsilon}^{j}}{\partial n} & =\gamma_{0} n_{j} \quad \text { on } \partial \Omega, \\
\int_{\partial \Omega} v_{\epsilon}^{j} d \sigma & =0
\end{aligned}\right.
$$

where $n_{j}$ is the $j$-th component of the unit normal direction $n$ to $\partial \Omega$.

The tensor $M$ is consistently defined in [7] as the following limit

$$
\int_{\Omega} M_{i j}(x) \phi(x) d \mu=\lim _{\epsilon \rightarrow 0} \frac{1}{\left|\omega_{\epsilon}\right|} \int_{\omega_{\epsilon}} \frac{\partial v_{\epsilon}^{j}}{\partial x_{i}}(x) \phi(x) d x,
$$

for every smooth function $\phi$.

We shall make use of the alternative equivalent definition ([8]).

Lemma 2.1. Let $M$ be the polarization tensor given by (6) and let $\phi$ be a positive smooth function on $\Omega$, then, for every direction $\xi \in \mathbb{R}^{3}$,

$$
\begin{aligned}
\int_{\Omega}\left(\gamma_{1}-\gamma_{0}\right) M \xi \cdot \xi \phi d \mu & =\frac{1}{\left|\omega_{\epsilon}\right|} \int_{\omega_{\epsilon}}\left(\gamma_{1}-\gamma_{0}\right) \frac{\gamma_{0}}{\gamma_{1}}|\xi|^{2} \phi d x \\
& +\frac{1}{\left|\omega_{\epsilon}\right|} \min _{w \in H^{1}(\Omega)} \int_{\Omega} \gamma_{\epsilon}\left|\nabla w+\mathbf{1}_{\omega_{\epsilon}} \frac{\gamma_{1}-\gamma_{0}}{\gamma_{1}} \xi\right|^{2} \phi d x+o(1) .
\end{aligned}
$$

where o(1) tends to zero with $\epsilon$.

\section{Polarization tensor for a Cylindrical inClusion in $\mathbb{R}^{3}$}

In this work we will consider cylindrical inclusions having fixed height and small basis. For sake of simplicity we will fix our coordinate system in the center of the cylinder and the third coordinate direction $\left(e_{3}\right)$ parallel to the axis of the cylinder. We will use the notation $x=\left(x_{1}, x_{2}, x_{3}\right)$. Consider an inclusion $\omega_{\epsilon}$ given by

$$
\omega_{\epsilon}=\omega_{2, \epsilon} \times(-l, l),
$$

where $\omega_{2, \epsilon}$ is a bidimensional measurable set.

We assume that, for each $\epsilon, \omega_{2, \epsilon} \subset D=D(0, r)$, the disk of radius $r$ centered at the origin and that, for some $L>l$ the cylinder $K_{0}=\bar{D} \times[-L, L]$ is contained in $\Omega$. We will also assume that $\lim _{\epsilon \rightarrow 0}\left|\omega_{2, \epsilon}\right|=0$ and

$$
\left|\omega_{2, \epsilon}\right|^{-1} 1_{\omega_{2, \epsilon}}(\cdot) \text { converges in the sense of measure to } \mu^{\prime} \text { when } \epsilon \rightarrow 0 \text {. }
$$

The Borel measure $\mu$ defined in (4) and $\mu^{\prime}$ are related by

$$
\int_{K_{0}} \psi d \mu=\frac{1}{2 l} \int_{-l}^{l} \int_{D} \psi d \mu^{\prime} d x_{3}, \quad \text { for each } \psi \in C\left(K_{0}\right) .
$$

Let $\gamma_{0}$ be the smooth conductivity of the material in $\Omega$ and let $\gamma_{1} \neq \gamma_{0}$ be the smooth conductivity of the inclusion (for sake of generality we assume that both $\gamma_{0}$ and $\gamma_{1}$ are defined in the whole body $\Omega$ ). The conductivity in the body containing the inclusion is given by (2). 


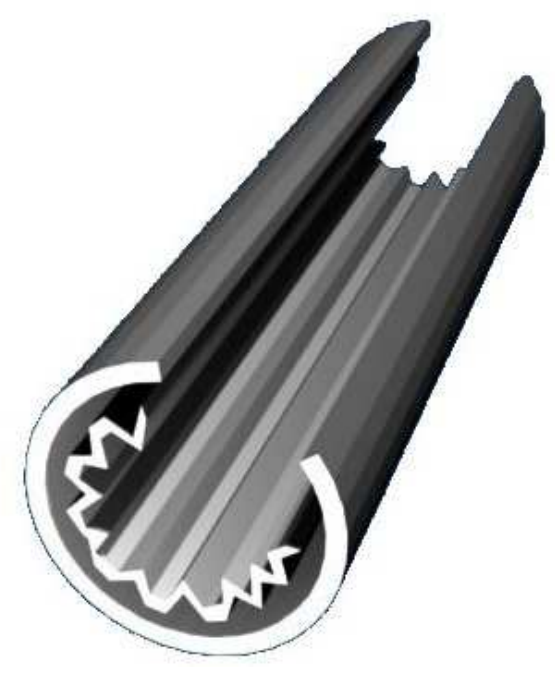

Figure 1. A cylindrical inclusion. The base of the cylinder $\omega_{2, \epsilon}$ has a small area.

Now let us slice our 3-dimensional body in sections that are parallel to the plane $\left\{x_{3}=0\right\}$. Let us denote by $\Omega_{x_{3}}=\left\{\left(x_{1}, x_{2}\right) \in \mathbb{R}^{2}:\left(x_{1}, x_{2}, x_{3}\right) \in \Omega\right\}$ each of this slices. Let us define

$$
\gamma_{2, \epsilon}(x)=\left(\gamma_{1}(x)-\gamma_{0}(x)\right) \mathbf{1}_{\omega_{2, \epsilon}}\left(x_{1}, x_{2}\right)+\gamma_{0}(x) .
$$

For $x_{3} \in(-l, l)$ this coefficient represents the conductivity of the slice $\Omega_{x_{3}}$. For each $x_{3}$, there is a $2 \times 2$ polarization tensor $m(x)$ that can be defined (as in Lemma 2.1) in the following way:

Lemma 3.1. Let $\phi$ be a positive smooth function in $\Omega$. Then, for every direction $\eta \in \mathbb{R}^{2}$,

$$
\begin{aligned}
\int_{\Omega_{x_{3}}}\left(\gamma_{1}-\gamma_{0}\right) & m \eta \cdot \eta \phi d \mu^{\prime}=\frac{1}{\left|\omega_{2, \epsilon}\right|} \int_{\omega_{2, \epsilon}}\left(\gamma_{1}-\gamma_{0}\right) \frac{\gamma_{0}}{\gamma_{1}}|\eta|^{2} \phi d x_{1} d x_{2} \\
& +\frac{1}{\left|\omega_{2, \epsilon}\right|} \min _{w \in H^{1}\left(\Omega_{x_{3}}\right)} \int_{\Omega_{x_{3}}} \gamma_{2, \epsilon}\left|\nabla w+\mathbf{1}_{\omega_{2, \epsilon}} \frac{\gamma_{1}-\gamma_{0}}{\gamma_{1}} \xi\right|^{2} \phi d x_{1} d x_{2}+o(1) .
\end{aligned}
$$

where o(1) tends to zero with $\epsilon$.

Notice that, although the measure $\mu^{\prime}$ is the same in each slice, the polarization tensor $m$ may change because both conductivities $\gamma_{0}$ and $\gamma_{1}$ depend on $x_{3}$.

Now, we state and prove the main results of this section.

Proposition 3.2. If $\omega_{\epsilon}$ is given by (8), the unit vector $e_{3}$ is an eigenvector for the polarization tensor $M(x)$, that is,

$$
M(x) e_{3} \cdot e_{3}=1 \quad \text { for } \quad \mu \text {-almost every } \quad x \in \Omega .
$$

Since the polarization tensor is symmetric (see [7]), this implies that there are other two eigenvectors in the orthogonal plane, the one spanned by $e_{1}$ and $e_{2}$.

We prove that, in that plane, the polarization tensor coincide with the 2-dimensional tensor.

Proposition 3.3. Let $\omega_{\epsilon}$ be given by (8) and let $m$ be the 2-dimensional polarization tensor defined by (9). Let $\eta$ be any direction in $\mathbb{R}^{2}$, and denote by $\eta^{*}$ its extension $\eta^{*}=(\eta, 0)$. Then,

$$
M(x) \eta^{*} \cdot \eta^{*}=m(x) \eta \cdot \eta \quad \text { for } \mu \text {-almost every } \quad x \in \Omega \text {. }
$$

In order to prove Propositions (3.2) and (3.3) we are going to use definitions (7) and (9) of polarization tensors. Before doing that, we need to point out a variant of those formulas that is justified by $[8$, remark 1, p.185]. According to this remark, the minimum in formula (7) need not to be taken over $H^{1}(\Omega)$, but it can be taken over $H_{0}^{1}\left(\Omega^{\prime}\right)$ for any convex set $\Omega^{\prime}$ that contains the 
whole family of inclusions. In our case we can choose $\Omega^{\prime}=K_{0}$. The same holds in formula (9) where the minimum can be taken over $H_{0}^{1}(D)$.

Let us fix a positive smooth function $\phi$ defined in $\Omega$ and, for $j=1,2,3$, let us denote by $\Phi_{\epsilon}^{j}$ the 3-dimensional minimizer in (7) corresponding to $\xi=e_{j}$. Each minimizer $\Phi_{\epsilon}^{j} \in H_{0}^{1}\left(K_{0}\right)$ is solution to

$$
\operatorname{div}\left(\gamma_{\epsilon} \phi \nabla \Phi_{\epsilon}^{j}\right)=\operatorname{div}\left(\left(\gamma_{0}-\gamma_{1}\right) \mathbf{1}_{\omega_{\epsilon}} e_{j} \phi\right) \text { in } K_{0} .
$$

By Lemma 5.1 in the Appendix, the minimizer $\Phi_{\epsilon}^{j}$ satisfies the estimates

$$
\left\|\nabla \Phi_{\epsilon}^{j}\right\|_{L^{2}\left(K_{0}\right)} \leq C\left|\omega_{\epsilon}\right|^{1 / 2}, \quad \text { for } \quad j=1,2,3,
$$

and

$$
\left\|\Phi_{\epsilon}^{j}\right\|_{L^{2}\left(K_{0}\right)} \leq C\left|\omega_{\epsilon}\right|^{1 / 2+\alpha}, \quad \text { for } \quad j=1,2,3,
$$

where the positive constants $C$ and $\alpha$ depend on $K_{0}, c_{0}$ and $\phi$, but not on $\epsilon$.

For the bidimensional tensor, we denote by $\psi_{\epsilon}^{j}$, for $j=1,2$, the functions in $H_{0}^{1}(D)$ defined by

$$
\operatorname{div}_{12}\left(\gamma_{2, \epsilon} \phi \nabla_{12} \psi_{\epsilon}^{j}\right)=\operatorname{div}_{12}\left(\left(\gamma_{0}-\gamma_{1}\right) \mathbf{1}_{\omega_{2, \epsilon}} e_{j} \phi\right) \text { in } D,
$$

where the notations $\operatorname{div}_{12}$ and $\nabla_{12}$ mean divergence and gradient with respect to the first two variables only. The third variable plays the role of a parameter. Due to Lemma 5.1 in the Appendix these functions satisfies the estimates

$$
\left\|\nabla_{12} \psi_{\epsilon}^{j}\right\|_{L^{2}(D)} \leq C\left|\omega_{2, \epsilon}\right|^{1 / 2}, \quad \text { for } \quad j=1,2,
$$

and

$$
\left\|\psi_{\epsilon}^{j}\right\|_{L^{2}(D)} \leq C\left|\omega_{2, \epsilon}\right|^{1 / 2+\alpha}, \quad \text { for } \quad j=1,2,
$$

where the positive constants $C$ and $\alpha$ depend on $K_{0}, c_{0}$ and $\phi$, but not on $\epsilon$.

Proof of Proposition 3.2. As it was noted in [8], it is easy to recover the optimal pointwise estimates of the polarization tensor $M$ from (7). namely that

$$
\min \left\{1, \frac{\gamma_{0}(x)}{\gamma_{1}(x)}\right\}|\xi|^{2} \leq M_{i j}(x) \xi_{i} \xi_{j} \leq \max \left\{1, \frac{\gamma_{0}(x)}{\gamma_{1}(x)}\right\}|\xi|^{2},
$$

for every $\xi \in \mathbb{R}^{3}$ and for $x \mu$-almost everywhere in $\Omega$.

As a consequence, showing (10) will ensure that 1 is either the maximal or minimal eigenvalue of $M$, with eigenvector $e_{3}$.

Let $\Phi_{\epsilon}^{3}$ be the minimizer corresponding to $\xi=e_{3}$.

Let us notice that $\Phi_{\epsilon}^{3}$ is a solution of (11) and, by De Giorgi-Nash estimates (see Theorem 8.24 in [11]), it is Hölder continuous and, for every $x \in K_{1} \subset \subset K_{0}$

$$
\left|\Phi_{\epsilon}^{3}(x)\right| \leq C\left(\left\|\Phi_{\epsilon}^{3}\right\|_{L^{2}\left(K_{0}\right)}+\left\|\mathbf{1}_{\omega_{\epsilon}} \psi\right\|_{L^{4}\left(K_{0}\right)}\right) .
$$

By (13) we deduce that

$$
\left|\Phi_{\epsilon}^{3}(x)\right| \leq C\left(\left|\omega_{\epsilon}\right|^{1 / 2+\alpha}+\left|\omega_{\epsilon}\right|^{1 / 4}\right) \leq C\left|\omega_{\epsilon}\right|^{1 / 4} .
$$

By definition (11) and integrating by parts,

$$
\begin{aligned}
\int_{K_{0}} \gamma_{\epsilon}\left|\nabla \Phi_{\epsilon}^{3}\right|^{2} \phi d x= & \int_{\omega_{\epsilon}}\left(\gamma_{0}-\gamma_{1}\right) \phi e_{3} \cdot \nabla \Phi_{\epsilon}^{3} d x \\
= & \int_{\omega_{2, \epsilon}} \int_{-l}^{l}\left(\gamma_{0}-\gamma_{1}\right) \phi \frac{\partial}{\partial x_{3}} \Phi_{\epsilon}^{3} d x_{3} d x_{1} d x_{2} \\
= & {\left[\int_{\omega_{2, \epsilon}}\left(\gamma_{0}-\gamma_{1}\right) \phi \Phi_{\epsilon}^{3} d x_{1} d x_{2}\right]_{x_{3}=-l}^{x_{3}=l} } \\
& -\int_{\omega_{\epsilon}} \Phi_{\epsilon}^{3} \frac{\partial}{\partial x_{3}}\left(\left(\gamma_{0}-\gamma_{1}\right) \phi\right) d x
\end{aligned}
$$


By (17) (noticing that $\omega_{2, \epsilon} \times[-l, l] \subset \subset K_{0}$ ) and since $\gamma_{0}, \gamma_{1}$ and $\phi$ are smooth, we get

$$
\left|\left[\int_{\omega_{2, \epsilon}}\left(\gamma_{0}-\gamma_{1}\right) \phi \Phi_{\epsilon}^{3} d x_{1} d x_{2}\right]_{x_{3}=-l}^{x_{3}=l}\right| \leq C\left|\omega_{\epsilon}\right|^{1 / 4}\left|\omega_{2, \epsilon}\right| \leq \frac{C}{l}\left|\omega_{\epsilon}\right|^{5 / 4}
$$

where $C$ does not depend on $\epsilon$ and on $l$.

In addition, by using Cauchy-Schwarz inequality, and estimate (13) we obtain

$$
\left|\int_{\omega_{\epsilon}} \Phi_{\epsilon}^{3}(x) \frac{\partial}{\partial x_{3}}\left(\left(\gamma_{0}-\gamma_{1}\right) \phi\right) d x\right| \leq C\left|\omega_{\epsilon}\right|^{1+\alpha}
$$

By putting together (18), (19) and (20), we get

$$
\int_{K_{0}} \gamma_{\epsilon}\left|\nabla \Phi_{\epsilon}^{3}\right|^{2} \phi d x \leq C\left|\omega_{\epsilon}\right|^{1+\alpha^{\prime}}, \quad \text { where } \quad \alpha^{\prime}=\min (1 / 4, \alpha) .
$$

Let us now write formula (7) for $\xi=e_{3}$ :

$$
\begin{aligned}
\int_{\Omega}\left(\gamma_{1}-\gamma_{0}\right) M e_{3} \cdot e_{3} \phi d \mu & =\frac{1}{\left|\omega_{\epsilon}\right|} \int_{\omega_{\epsilon}}\left(\gamma_{1}-\gamma_{0}\right) \frac{\gamma_{0}}{\gamma_{1}} \phi d x \\
& +\frac{1}{\left|\omega_{\epsilon}\right|} \int_{K_{0}} \gamma_{\epsilon}\left|\nabla \Phi_{\epsilon}^{3}+\mathbf{1}_{\omega_{\epsilon}} \frac{\gamma_{1}-\gamma_{0}}{\gamma_{1}} e_{3}\right|^{2} \phi d x+o(1) \\
& =\frac{1}{\left|\omega_{\epsilon}\right|} \int_{\omega_{\epsilon}}\left(\gamma_{1}-\gamma_{0}\right) \phi d x+\frac{1}{\left|\omega_{\epsilon}\right|} \int_{K_{0}} \gamma_{\epsilon}\left|\nabla \Phi_{\epsilon}^{3}\right|^{2} \phi d x \\
& +\frac{2}{\left|\omega_{\epsilon}\right|} \int_{K_{0}} \mathbf{1}_{\omega_{\epsilon}} \frac{\gamma_{\epsilon}\left(\gamma_{1}-\gamma_{0}\right)}{\gamma_{1}} \phi e_{3} \cdot \nabla \Phi_{\epsilon}^{3} d x+o(1) .
\end{aligned}
$$

By Cauchy-Schwarz, and (21)

$$
\left|\frac{2}{\left|\omega_{\epsilon}\right|} \int_{K_{0}} \mathbf{1}_{\omega_{\epsilon}} \frac{\gamma_{\epsilon}\left(\gamma_{1}-\gamma_{0}\right)}{\gamma_{1}} \phi e_{3} \cdot \nabla \Phi_{\epsilon}^{3} d x\right| \leq C\left|\omega_{\epsilon}\right|^{\alpha^{\prime} / 2} .
$$

By inserting (23) and (21) into (22) we get

$$
\int_{\Omega}\left(\gamma_{1}-\gamma_{0}\right) M e_{3} \cdot e_{3} \phi d \mu=\frac{1}{\left|\omega_{\epsilon}\right|} \int_{\omega_{\epsilon}}\left(\gamma_{1}-\gamma_{0}\right) \phi d x+o(1),
$$

and, by letting $\epsilon \rightarrow 0$, we get

$$
\int_{\Omega}\left(\gamma_{1}-\gamma_{0}\right) M e_{3} \cdot e_{3} \phi d \mu=\int_{\Omega}\left(\gamma_{1}-\gamma_{0}\right) \phi d \mu
$$

which, in turn, implies (10).

Proof of Proposition 3.3. The idea of the proof consists in constructing an approximation of the correctors $\Phi_{\epsilon}^{1}$ and $\Phi_{\epsilon}^{2}$ by using the 2-dimensional correctors $\psi_{\epsilon}^{1}$ and $\psi_{\epsilon}^{2}$ defined by (14).

Let $f_{\epsilon}\left(x_{3}\right)$ be a function of $x_{3}$ only that we will specify better in Lemma 3.6. Let us define, for $j=1,2$,

$$
\tilde{\Phi}_{\epsilon}^{j}(x)=\psi_{\epsilon}^{j}(x) f_{\epsilon}\left(x_{3}\right) \quad \text { for } \quad x \in K_{0} .
$$

Our proof will make use of the following technical results:

Lemma 3.4. For $j=1,2$, the functions $\psi_{\epsilon}^{j}$ satisfies

$$
\left\|\frac{\partial}{\partial x_{3}} \psi_{\epsilon}^{j}\right\|_{L^{2}\left(K_{0}\right)} \leq C\left|\omega_{2, \epsilon}\right|^{\frac{1}{2}+\alpha}
$$

for some positive $C$ and $\alpha$ independent of $\epsilon$ and of $l$. 
Lemma 3.5. Let us denote by $\mathbf{1}_{l}\left(x_{3}\right)=\mathbf{1}_{(-l, l)}\left(x_{3}\right)$. Assume $f_{\epsilon} \in H^{1}(\mathbb{R})$ is chosen so that

$$
\begin{gathered}
0 \leq f_{\epsilon} \leq 1 \quad \text { and } \quad f_{\epsilon}\left(x_{3}\right) \mathbf{1}_{l}\left(x_{3}\right)=\mathbf{1}_{l}\left(x_{3}\right), \\
\left\|f_{\epsilon}^{\prime}\right\|_{L^{2}(-L, L)} \leq C\left|\omega_{2, \epsilon}\right|^{-\frac{\alpha}{2}} \\
\left\|f_{\epsilon}(\cdot)\left(1-\mathbf{1}_{l}(\cdot)\right)\right\|_{L^{2}(-L, L)} \leq C\left|\omega_{2, \epsilon}\right|^{\frac{\alpha}{2}}
\end{gathered}
$$

Then, for $j=1,2$, the functions $\tilde{\Phi}_{\epsilon}^{j}$ and $\Phi_{\epsilon}^{j}$, given by (24) and (11), respectively, satisfy the inequality

$$
\left\|\nabla\left(\tilde{\Phi}_{\epsilon}^{j}-\Phi_{\epsilon}^{j}\right)\right\|_{L^{2}\left(K_{0}\right)} \leq C\left|\omega_{2, \epsilon}\right|^{\frac{1+\alpha}{2}},
$$

where $C$ and $\alpha$ are independent of $\epsilon$.

Lemma 3.6. The function $f_{\epsilon}$ given by

$$
f_{\epsilon}\left(x_{3}\right)=\left\{\begin{array}{cll}
0 & \text { if } & x_{3}<-l-2\left|\omega_{2, \epsilon}\right|^{\alpha} \\
\frac{\left(x_{3}+l+2\left|\omega_{2, \epsilon}\right|^{\alpha}\right)^{2}}{2\left|\omega_{2, \epsilon}\right|^{2 \alpha}} & \text { if } & x_{3} \in\left[-l-2\left|\omega_{2, \epsilon}\right|^{\alpha},-l-\left|\omega_{2, \epsilon}\right|^{\alpha}\right] \\
1-\frac{\left(x_{3}+l\right)^{2}}{2\left|\omega_{2, \epsilon}\right|^{2 \alpha}} & \text { if } & x_{3} \in\left[-l-\left|\omega_{2, \epsilon}\right|^{\alpha},-l\right] \\
1 & \text { if } & x_{3} \in[-l, 0],
\end{array}\right.
$$

and such that $f_{\epsilon}\left(-x_{3}\right)=f_{\epsilon}\left(x_{3}\right)$, satisfies assumptions (26), (27) and (28).

The proof of Lemma 3.6 is safely left to the reader. Lemma 3.4 and Lemma 3.5 are proven below. Let us first proceed with the proof of Proposition 3.3.

Let us take $\eta^{*}=(\eta, 0)$ where $\eta$ is a unit vector in $\mathbb{R}^{2}$. Let us consider formula (7) for $\xi=\eta^{*}$ and with the minimum taken over $H_{0}^{1}\left(K_{0}\right)$. We write the minimizer $w_{\epsilon}=\sum_{j=1}^{2} \eta_{j} \Phi_{\epsilon}^{j}$ as $w_{\epsilon}=$ $\sum_{j=1}^{2} \eta_{j} \tilde{\Phi}_{\epsilon}^{j}+\sum_{j=1}^{2} \eta_{j}\left(\Phi_{\epsilon}^{j}-\tilde{\Phi}_{\epsilon}^{j}\right)$ and obtain

$$
\begin{aligned}
\int_{\Omega}\left(\gamma_{1}-\gamma_{0}\right) M \eta^{*} \cdot \eta^{*} \phi d \mu & =\int_{\Omega}\left(\gamma_{1}-\gamma_{0}\right) \frac{\gamma_{0}}{\gamma_{1}} \phi d \mu \\
& +\frac{1}{\left|\omega_{\epsilon}\right|} \int_{K_{0}} \gamma_{\epsilon}\left|\nabla\left(\sum_{j=1}^{2} \eta_{j} \tilde{\Phi}_{\epsilon}^{j}\right)+\frac{\gamma_{1}-\gamma_{0}}{\gamma_{1}} \mathbf{1}_{\omega_{\epsilon}} \eta^{*}\right|^{2} \phi d x \\
& +r_{1, \epsilon}+r_{2, \epsilon}
\end{aligned}
$$

where we have set

$$
r_{1, \epsilon}=\frac{1}{\left|\omega_{\epsilon}\right|} \int_{K_{0}} \gamma_{\epsilon}\left|\nabla\left(\sum_{j=1}^{2} \eta_{j}\left(\Phi_{\epsilon}^{j}-\tilde{\Phi}_{\epsilon}^{j}\right)\right)\right|^{2} \phi d x
$$

and

$$
r_{2, \epsilon}=\frac{2}{\left|\omega_{\epsilon}\right|} \int_{K_{0}} \gamma_{\epsilon}\left(\nabla\left(\sum_{j=1}^{2} \eta_{j} \tilde{\Phi}_{\epsilon}^{j}\right)+\frac{\gamma_{1}-\gamma_{0}}{\gamma_{1}} \mathbf{1}_{\omega_{\epsilon}} \eta^{*}\right) \cdot \nabla\left(\sum_{j=1}^{2} \eta_{j}\left(\Phi_{\epsilon}^{j}-\tilde{\Phi}_{\epsilon}^{j}\right)\right) \phi d x .
$$

Let us first show that $r_{1, \epsilon}$ and $r_{2, \epsilon}$ are small. The term $r_{1, \epsilon}$ can be estimated by Lemma 3.5, so that

$$
\left|r_{1, \epsilon}\right| \leq \frac{C}{\left|\omega_{\epsilon}\right|} \sum_{j=1}^{2}\left\|\nabla\left(\Phi_{\epsilon}^{j}-\tilde{\Phi}_{\epsilon}^{j}\right)\right\|_{L^{2}\left(K_{0}\right)}^{2} \leq \frac{C}{l}\left|\omega_{2, \epsilon}\right|^{\alpha} .
$$


For $r_{2, \epsilon}$, using Cauchy-Schwarz inequality and Lemma 3.5, we obtain

$$
\begin{aligned}
\left|r_{2, \epsilon}\right| & \leq \frac{C}{\left|\omega_{\epsilon}\right|}\left|\omega_{2, \epsilon}\right|^{\frac{1+\alpha}{2}}\left\|\nabla\left(\sum_{j=1}^{2} \eta_{j} \tilde{\Phi}_{\epsilon}^{j}\right)+\frac{\gamma_{1}-\gamma_{0}}{\gamma_{1}} \mathbf{1}_{\omega_{\epsilon}} \eta^{*}\right\|_{L^{2}\left(K_{0}\right)} \\
& \leq \frac{C}{l}\left|\omega_{2, \epsilon}\right|^{\frac{\alpha-1}{2}}\left(\left\|\nabla\left(\sum_{j=1}^{2} \eta_{j} \tilde{\Phi}_{\epsilon}^{j}\right)\right\|_{L^{2}\left(K_{0}\right)}+\left|\omega_{\epsilon}\right|^{\frac{1}{2}}\right) .
\end{aligned}
$$

Let us note that

$$
\left\|\nabla \tilde{\Phi}_{\epsilon}^{j}\right\|_{L^{2}\left(K_{0}\right)} \leq\left\|f_{\epsilon} \nabla_{12} \psi_{\epsilon}^{j}\right\|_{L^{2}\left(K_{0}\right)}+\left\|f_{\epsilon}^{\prime} \psi_{\epsilon}^{j}+f_{\epsilon} \frac{\partial}{\partial x_{3}} \psi_{\epsilon}^{j}\right\|_{L^{2}\left(K_{0}\right)} .
$$

Moreover, by (16) we get

$$
\begin{aligned}
\left\|f_{\epsilon} \nabla_{12} \psi_{\epsilon}^{j}\right\|_{L^{2}\left(K_{0}\right)} & =\left(\int_{-L}^{L} \int_{D} f_{\epsilon}^{2}\left|\nabla_{12} \psi_{\epsilon}^{j}\right|^{2} d x_{1} d x_{2} d x_{3}\right)^{\frac{1}{2}} \\
& \leq 2 L\left\|\nabla_{12} \psi_{\epsilon}^{j}\right\|_{L^{2}(D)} \leq C\left|\omega_{2, \epsilon}\right|^{\frac{1}{2}}
\end{aligned}
$$

by (25) and (27), we obtain

$$
\left\|\psi_{\epsilon}^{j} f_{\epsilon}^{\prime}\right\|_{L^{2}\left(K_{0}\right)} \leq C\left|\omega_{2, \epsilon}\right|^{\frac{1}{2}+\alpha}\left|\omega_{2, \epsilon}\right|^{-\frac{\alpha}{2}}
$$

and, by Lemma 3.4

$$
\left\|f_{\epsilon} \frac{\partial}{\partial x_{3}} \psi_{\epsilon}^{j}\right\|_{L^{2}\left(K_{0}\right)} \leq C\left|\omega_{2, \epsilon}\right|^{\frac{1}{2}+\alpha}
$$

Hence, finally

$$
\left|r_{2, \epsilon}\right| \leq \frac{C}{l}\left|\omega_{2, \epsilon}\right|^{\frac{\alpha-1}{2}}\left(\left|\omega_{2, \epsilon}\right|^{\frac{1}{2}}+\left|\omega_{\epsilon}\right|^{\frac{1}{2}}\right) \leq C\left|\omega_{2, \epsilon}\right|^{\frac{\alpha}{2}} \frac{1}{l}
$$

Now, we consider the second term of the right-hand-side in (29):

$$
\begin{aligned}
& \frac{1}{\left|\omega_{\epsilon}\right|} \int_{K_{0}} \gamma_{\epsilon}\left|\nabla\left(\sum_{j} \eta_{j} \tilde{\Phi}_{\epsilon}^{j}\right)+\frac{\gamma_{1}-\gamma_{0}}{\gamma_{1}} \mathbf{1}_{\omega_{\epsilon}} \eta^{*}\right|^{2} \phi d x \\
& =\frac{1}{\left|\omega_{\epsilon}\right|} \int_{-l}^{l} \int_{D} \gamma_{\epsilon}\left|\sum_{j} \eta_{j} \nabla_{12} \psi_{\epsilon}^{j} f_{\epsilon}+\frac{\gamma_{1}-\gamma_{0}}{\gamma_{1}} \mathbf{1}_{\omega_{2, \epsilon}} \eta\right|^{2} \phi d x+ \\
& +\frac{1}{\left|\omega_{\epsilon}\right|} \int_{-L}^{L}\left(1-\mathbf{1}_{l}\left(x_{3}\right)\right) \int_{D} \gamma_{\epsilon}\left|\sum_{j} \eta_{j} \nabla_{12} \psi_{\epsilon}^{j} f_{\epsilon}\right|^{2} \phi d x \\
& +\frac{1}{\left|\omega_{\epsilon}\right|} \int_{K_{0}} \gamma_{\epsilon}\left[\frac{\partial}{\partial x_{3}}\left(\sum_{j} \eta_{j} \psi_{\epsilon}^{j}\right) f_{\epsilon}+\sum_{j} \eta_{j} \psi_{\epsilon}^{j} f_{\epsilon}^{\prime}\right]^{2} \phi d x .
\end{aligned}
$$


Let us notice that, for $x_{3} \in(-l, l), f_{\epsilon}\left(x_{3}\right)=1$ and, by $(9)$,

$$
\begin{aligned}
& \frac{1}{\left|\omega_{\epsilon}\right|} \int_{-l}^{l} \int_{D} \gamma_{\epsilon}\left|\sum_{j} \eta_{j} \nabla_{12} \psi_{\epsilon}^{j} f_{\epsilon}+\frac{\gamma_{1}-\gamma_{0}}{\gamma_{1}} \mathbf{1}_{\omega_{2, \epsilon}} \eta\right|^{2} \phi d x \\
& =\frac{1}{2 l\left|\omega_{2, \epsilon}\right|} \int_{-l}^{l} \int_{D} \gamma_{2, \epsilon}\left|\sum_{j} \eta_{j} \nabla_{12} \psi_{\epsilon}^{j}+\frac{\gamma_{1}-\gamma_{0}}{\gamma_{1}} \mathbf{1}_{\omega_{2, \epsilon}} \eta\right|^{2} \phi d x \\
& =\frac{1}{2 l} \int_{-l}^{l} \int_{D}\left(\gamma_{1}-\gamma_{0}\right) m \eta \cdot \eta \phi d \mu^{\prime} d x_{3}-\frac{1}{2 l\left|\omega_{2, \epsilon}\right|} \int_{-l}^{l} \int_{\omega_{2, \epsilon}}\left(\gamma_{1}-\gamma_{0}\right) \frac{\gamma_{0}}{\gamma_{1}} \phi d x+o(1) \\
& =\int_{\Omega}\left(\gamma_{1}-\gamma_{0}\right) m \eta \cdot \eta \phi d \mu-\int_{\Omega}\left(\gamma_{1}-\gamma_{0}\right) \frac{\gamma_{0}}{\gamma_{1}} \phi d \mu+o(1) .
\end{aligned}
$$

Moreover, by (15) and (28),

$$
\begin{aligned}
& \frac{1}{\left|\omega_{\epsilon}\right|} \int_{-L}^{L}\left(1-\mathbf{1}_{l}\left(x_{3}\right)\right) \int_{D} \gamma_{0}\left|\sum_{j} \eta_{j} \nabla_{12} \psi_{\epsilon}^{j} f_{\epsilon}\right|^{2} \phi d x \\
& \leq \frac{C}{\left|\omega_{\epsilon}\right|}\left(\sum_{j}\left\|\nabla_{12} \psi_{\epsilon}^{j}\right\|_{L^{2}(D)}^{2}\left\|\left(1-\mathbf{1}_{l}\right) f_{\epsilon}\right\|_{L^{2}(-L, L)}^{2}\right) \\
& \leq \frac{C}{l}\left|\omega_{2, \epsilon}\right|^{\alpha}
\end{aligned}
$$

and, by Lemma 3.4, (16) and (27),

$$
\begin{aligned}
& \frac{1}{\left|\omega_{\epsilon}\right|} \int_{K_{0}} \gamma_{\epsilon}\left[\frac{\partial}{\partial x_{3}}\left(\sum_{j} \eta_{j} \psi_{\epsilon}^{j}\right) f_{\epsilon}+\sum_{j} \eta_{j} \psi_{\epsilon}^{j} f_{\epsilon}^{\prime}\right]^{2} \phi d x \\
& \leq \frac{C}{\left|\omega_{\epsilon}\right|}\left(\left\|\frac{\partial}{\partial x_{3}} \psi_{\epsilon}^{j}\right\|_{L^{2}\left(K_{0}\right)}^{2}+\left\|\psi_{\epsilon}^{j}\right\|_{L^{2}\left(K_{0}\right)}^{2}\left\|f_{\epsilon}^{\prime}\right\|_{L^{2}(-L, L)}^{2}\right) \\
& \leq \frac{C}{\left|\omega_{\epsilon}\right|}\left(\left|\omega_{2, \epsilon}\right|^{1+2 \alpha}+\left|\omega_{2, \epsilon}\right|^{1+2 \alpha}\left|\omega_{2, \epsilon}\right|^{-\alpha}\right)=\frac{C}{l}\left|\omega_{2, \epsilon}\right|^{\alpha} .
\end{aligned}
$$

As a result, identity (29) becomes

$$
\int_{\Omega}\left(\gamma_{1}-\gamma_{0}\right) M \eta^{*} \cdot \eta^{*} \phi d \mu=\int_{\Omega}\left(\gamma_{1}-\gamma_{0}\right) m \eta \cdot \eta \phi d \mu+o(1),
$$

that, passing to the limit for $\epsilon \rightarrow 0$, is our thesis.

Remark 3.7. In the proof of Proposition 3.3, we underlined the dependence of the estimates upon $l$, the macroscopic length of the cylinder. Truly, we make no use of that information. This is merely a reminder of the fact that our approach cannot be used directly for arbitray shapes: when $l$ tends to zero, these estimates become trivial.

Proof of Lemma 3.4. Let us fix $j=1$ or 2. If we differentiate equation (14) with respect to $x_{3}$, we obtain that for each $x_{3}, \frac{\partial}{\partial x_{3}} \psi_{\epsilon}^{j}$ satisfies equation

$$
\begin{aligned}
\operatorname{div}_{12}\left(\gamma_{\epsilon} \phi \nabla_{12}\left(\frac{\partial}{\partial x_{3}} \psi_{\epsilon}^{j}\right)\right)= & \operatorname{div}_{12}\left(\frac{\partial}{\partial x_{3}}\left(\left(\gamma_{0}-\gamma_{1}\right) \phi\right) \mathbf{1}_{\omega_{2, \epsilon}} e_{j}\right) \\
& -\operatorname{div}_{12}\left(\left(\frac{\partial}{\partial x_{3}}\left(\gamma_{\epsilon} \phi\right)\right) \nabla_{12} \psi_{\epsilon}^{j}\right)
\end{aligned}
$$


hence we can write

$$
\frac{\partial}{\partial x_{3}} \psi_{\epsilon}^{j}=a_{1, \epsilon}^{j}+a_{2, \epsilon}^{j}
$$

where for $i=1,2$, the function $a_{i, \epsilon}^{j} \in H_{0}^{1}(D)$ is the solution of

$$
\operatorname{div}_{12}\left(\gamma_{12}^{\epsilon} \phi \nabla_{12} a_{i, \epsilon}^{j}\right)=f_{i, \epsilon}^{j} \text { in } \quad D
$$

and where

$$
f_{1, \epsilon}^{j}=\operatorname{div}_{12}\left(\mathbf{1}_{\omega_{2, \epsilon}} e_{j} \frac{\partial}{\partial x_{3}}\left(\left(\gamma_{0}-\gamma_{1}\right) \phi\right)\right), f_{2, \epsilon}^{j}=-\operatorname{div}_{12}\left(\left(\frac{\partial}{\partial x_{3}}\left(\gamma_{\epsilon} \phi\right)\right) \nabla_{12} \psi_{\epsilon}^{j}\right) .
$$

We shall show that $a_{i, \epsilon}^{j}$ can be bounded as required. Concerning $a_{1, \epsilon}^{j}$, we can rely on Lemma 5.1 to obtain

$$
\left\|a_{1, \epsilon}^{j}\right\|_{L^{2}(D)} \leq C\left|\omega_{2, \epsilon}\right|^{1 / 2+\alpha} .
$$

Let us now turn to $a_{2, \epsilon}^{j}$. Notice that we can write

$$
\begin{aligned}
\operatorname{div}_{12}\left(\gamma_{12}^{\epsilon} \phi \nabla_{12} a_{2, \epsilon}^{j}\right) & =-\operatorname{div}_{12}\left(\frac{\partial}{\partial x_{3}}\left(\gamma_{12}^{\epsilon} \phi\right) \nabla_{12} \psi_{\epsilon}^{j}\right) \\
& =-\operatorname{div}_{12}\left(\left(\gamma_{12}^{\epsilon} \phi\right) \frac{\partial}{\partial x_{3}}\left(\log \left(\gamma_{12}^{\epsilon} \phi\right)\right) \nabla_{12} \psi_{\epsilon}^{j}\right) \\
& =-\operatorname{div}_{12}\left(\left(\gamma_{12}^{\epsilon} \phi\right) \nabla_{12}\left(\left(\frac{\partial}{\partial x_{3}}\left(\log \left(\gamma_{12}^{\epsilon} \phi\right)\right) \psi_{\epsilon}^{j}\right)\right)\right) \\
& +\operatorname{div}_{12}\left(\left(\gamma_{12}^{\epsilon} \phi\right) \psi_{\epsilon}^{j} \nabla_{12}\left(\frac{\partial}{\partial x_{3}}\left(\log \left(\gamma_{12}^{\epsilon} \phi\right)\right)\right)\right) .
\end{aligned}
$$

And this means that

$$
a_{2, \epsilon}^{j}=-\psi_{\epsilon}^{j} \frac{\partial}{\partial x_{3}}\left(\log \left(\gamma_{12}^{\epsilon} \phi\right)\right)+b_{\epsilon}^{j}
$$

with

$$
\operatorname{div}_{12}\left(\gamma_{12}^{\epsilon} \phi \nabla_{12} b_{\epsilon}^{j}\right)=\operatorname{div}_{12}\left(\left(\gamma_{12}^{\epsilon} \phi\right) \psi_{\epsilon}^{j} \nabla_{12}\left(\frac{\partial}{\partial x_{3}}\left(\log \left(\gamma_{12}^{\epsilon} \phi\right)\right)\right)\right) .
$$

By standard energy estimates, and by (16) we can conclude that

$$
\left\|\nabla_{12} b_{\epsilon}^{j}\right\|_{L^{2}(D)} \leq C\left|\omega_{2, \epsilon}\right|^{\frac{1}{2}+\alpha} .
$$

By Poincaré estimates for $b_{\epsilon}^{j}$, by (30) and (16) again, we can conclude that

$$
\left\|\nabla_{12} a_{2, \epsilon}^{j}\right\|_{L^{2}(D)} \leq C\left|\omega_{2, \epsilon}\right|^{\frac{1}{2}+\alpha} \text {. }
$$

Proof of Lemma 3.5. By (24) and (14), for $j=1,2$, given any function $\Psi \in H_{0}^{1}\left(K_{0}\right)$, we have

$$
\begin{aligned}
\int_{K_{0}} \gamma_{\epsilon} \phi \nabla \tilde{\Phi}_{\epsilon}^{j} \nabla \Psi d x & =\int_{K_{0}} \gamma_{\epsilon} \phi \nabla\left(f_{\epsilon} \psi_{\epsilon}^{j}\right) \nabla \Psi d x \\
=\int_{K_{0}} \gamma_{\epsilon} \phi f_{\epsilon} \nabla_{12} \psi_{\epsilon}^{j} \nabla_{12} \Psi d x & +\int_{K_{0}} \gamma_{\epsilon} \phi\left(f_{\epsilon}^{\prime} \psi_{\epsilon}^{j}+f_{\epsilon} \frac{\partial}{\partial x_{3}} \psi_{\epsilon}^{j}\right) \frac{\partial}{\partial x_{3}} \Psi d x \\
=\int_{\omega_{\epsilon}}\left(\gamma_{0}-\gamma_{1}\right) \phi \frac{\partial}{\partial x_{j}} \Psi d x & +\int_{K_{0}}\left(1-\mathbf{1}_{l}\right) \gamma_{\epsilon} \phi f_{\epsilon} \nabla_{12} \psi_{\epsilon}^{j} \nabla_{12} \Psi d x \\
& +\int_{K_{0}} \gamma_{\epsilon} \phi\left(f_{\epsilon}^{\prime} \psi_{\epsilon}^{j}+f_{\epsilon} \frac{\partial}{\partial x_{3}} \psi_{\epsilon}^{j}\right) \frac{\partial}{\partial x_{3}} \Psi d x
\end{aligned}
$$

while, by (11),

$$
\int_{K_{0}} \gamma_{\epsilon} \phi \nabla \Phi_{\epsilon}^{j} \nabla \Psi d x=\int_{\omega_{\epsilon}}\left(\gamma_{0}-\gamma_{1}\right) \phi \frac{\partial}{\partial x_{j}} \Psi d x
$$


and, hence,

$$
\begin{aligned}
& \int_{K_{0}} \gamma_{\epsilon} \phi\left(\nabla \Phi_{\epsilon}^{j}-\nabla \tilde{\Phi}_{\epsilon}^{j}\right) \nabla \Psi d x= \\
& \int_{K_{0}}\left(1-\mathbf{1}_{l}\right) \gamma_{\epsilon} \phi f_{\epsilon} \nabla_{12} \psi_{\epsilon}^{j} \nabla_{12} \Psi d x+\int_{K_{0}} \gamma_{\epsilon} \phi\left(f_{\epsilon}^{\prime} \psi_{\epsilon}^{j}+f_{\epsilon} \frac{\partial}{\partial x_{3}} \psi_{\epsilon}^{j}\right) \frac{\partial}{\partial x_{3}} \Psi d x
\end{aligned}
$$

from which it follows that

$$
\begin{aligned}
& \left\|\nabla \Phi_{\epsilon}^{j}-\nabla \tilde{\Phi}_{\epsilon}^{j}\right\|_{L^{2}\left(K_{0}\right)} \\
& \leq\left\|\nabla_{12} \psi_{\epsilon}^{j}\right\|_{L^{2}(D)}\left\|\left(1-\mathbf{1}_{l}\right) f_{\epsilon}\right\|_{L^{2}(-L, L)}+\left\|f_{\epsilon}^{\prime} \psi_{\epsilon}^{j}\right\|_{L^{2}\left(K_{0}\right)}+\left\|f_{\epsilon} \frac{\partial}{\partial x_{3}} \psi_{\epsilon}^{j}\right\|_{L^{2}\left(K_{0}\right)} \\
& \leq C\left(\left|\omega_{2, \epsilon}\right|^{\frac{1}{2}}\left|\omega_{2, \epsilon}\right|^{\frac{\alpha}{2}}+\left|\omega_{2, \epsilon}\right|^{\frac{1}{2}+\alpha}\left|\omega_{2, \epsilon}\right|^{-\frac{\alpha}{2}}+\left|\omega_{2, \epsilon}\right|^{\frac{1}{2}+\alpha}\right) \leq C\left|\omega_{2, \epsilon}\right|^{\frac{1+\alpha}{2}}
\end{aligned}
$$

\section{Reconstruction of the aXis of the Cylinder from Boundary Data of the CORRECTION TERM}

Let $\omega_{\epsilon}$ be a cylinder whose axis is a segment $\sigma \subset \subset \Omega$ and whose basis can be written as $\omega_{2, \epsilon}=\epsilon \omega_{2}$, where $\omega_{2}$ is a bidimensional domain of measure $\left|\omega_{2}\right|=1$.

Denote by $m$ the polarization tensor for $\epsilon \omega_{2}$ as defined in Lemma 3.1. From Propositions 3.2 and 3.3, it follows that, for $y \in \partial \Omega$,

$$
\begin{aligned}
\left(u_{\epsilon}-u_{0}\right)(y) & =\epsilon^{2} \int_{\sigma}\left(\gamma_{1}-\gamma_{0}\right)(x)\left[\frac{\partial u_{0}}{\partial \tau}(x) \frac{\partial N}{\partial \tau}(x, y)+\right. \\
& \left.+m(x) \widetilde{\nabla u_{0}}(x) \cdot \widetilde{\nabla N}(x, y)\right] d \sigma_{x}+o\left(\epsilon^{2}\right)
\end{aligned}
$$

where $\tau$ is the tangent direction to $\sigma$ and, for any vector $v \in \mathbb{R}^{3}$ we denote by $\tilde{v}=v-(v \cdot \tau) \tau$ the non tangential part of $v$.

Let us denote by $u_{\sigma}$ the function

$$
u_{\sigma}(y)=\int_{\sigma}\left(\gamma_{1}-\gamma_{0}\right)(x)\left[\frac{\partial u_{0}}{\partial \tau}(x) \frac{\partial N}{\partial \tau}(x, y)+m(x) \widetilde{\nabla u_{0}}(x) \cdot \widetilde{\nabla N}(x, y)\right] d \sigma_{x},
$$

defined for $y \in \Omega \backslash \sigma$.

In this section we want to address the following problem: do the boundary values of the correction term $u_{\sigma}$ uniquely determine the segment $\sigma$ ?

In order to answer to this question let us focus on some properties of this correction term.

First of all we observe that $u_{\sigma}$ is solution to

$$
\operatorname{div}\left(\gamma_{0} \nabla u_{\sigma}(x)\right)=0 \quad \text { for } \quad x \in \Omega \backslash \bar{\sigma} .
$$

Moreover

$$
\gamma_{0} \frac{\partial u_{\sigma}}{\partial n}=0 \quad \text { on } \quad \partial \Omega
$$

because $u_{\epsilon}$ and $u_{0}$ have the same conormal derivative on $\partial \Omega$.

If we integrate by parts in equation (31), and denote by $P$ and $Q$ the endpoints of segment $\sigma$ (such that $\tau=(Q-P) /|Q-P|$ ) we have

$$
\begin{aligned}
u_{\sigma}(y) & =N(Q, y)\left(\left(\gamma_{1}-\gamma_{0}\right) \frac{\partial u_{0}}{\partial \tau}(Q)\right)-N(P, y)\left(\left(\gamma_{1}-\gamma_{0}\right) \frac{\partial u_{0}}{\partial \tau}(P)\right) \\
& -\int_{\sigma} N(x, y) \frac{\partial}{\partial \tau}\left(\left(\gamma_{1}-\gamma_{0}\right) \frac{\partial u_{0}}{\partial \tau}\right) d \sigma_{x} \\
& +\int_{\sigma}\left(\gamma_{1}-\gamma_{0}\right) m(x) \widetilde{\nabla u_{0}}(x) \cdot \widetilde{\nabla N}(x, y) d \sigma_{x} .
\end{aligned}
$$


Although the formulation of the correction term $u_{\sigma}$ may look similar to the one that was established for inclusions that are small neighborhood of a curve in the plane (see [1]), we point out that the correction term given by (34) is singular at every point of the segment $\sigma$. Moreover, as it will be clear from the proof of the following proposition, at the endpoints of the segment $\sigma$, the correction term does not have worse singularities than at the other point of the segment. This behavior is different from the case analized in [1] where the correction term presents stronger singularities at the endpoints of the segment than at any other point.

Proposition 4.1. Let $\gamma_{0}$ and $\gamma_{1}$ be smooth positive functions. Let $\Sigma$ be an open subset of $\partial \Omega$ and let $\sigma$ and $\sigma^{\prime}$ be two segments strictly contained in $\Omega$. Let $u_{0}$ be a smooth solution to div $\left(\gamma_{0} \nabla u_{0}\right)=0$ in $\Omega$ such that $\nabla u_{0} \neq 0$ in $\Omega$, and let $u_{\sigma}$ and $u_{\sigma^{\prime}}$ be defined by (31) for segments $\sigma$ and $\sigma^{\prime}$ respectively.

$$
u_{\sigma}=u_{\sigma^{\prime}} \quad \text { on } \Sigma,
$$

then

$$
\sigma=\sigma^{\prime}
$$

Proof. For sake of simplicity, let us carry out the proof in the case of a constant conductivity $\gamma_{0}$. The general case is briefly discussed at the end.

Let $w=u_{\sigma}-u_{\sigma^{\prime}}$. By $(32)$, function $w$ is solution to

$$
\operatorname{div}\left(\gamma_{0} \nabla w\right)=0 \quad \text { in } \Omega \backslash\left(\bar{\sigma} \cup \overline{\sigma^{\prime}}\right) .
$$

Moreover, by (33) and (35), $w$ has zero Cauchy data on $\Sigma$, hence, by unique continuation property

$$
w \equiv 0 \quad \text { on } \quad \Omega \backslash\left(\bar{\sigma} \cup \overline{\sigma^{\prime}}\right) .
$$

We argue by contradiction and assume that $\sigma \neq \sigma^{\prime}$. This means that there is an endpoint, say $P$, that belongs to $\sigma$ but not to $\sigma^{\prime}$. Of course, this means that there is a segment $\gamma$ with endpoint in $P$ that belong to $\sigma \backslash \sigma^{\prime}$. We fix at $P$ the origin of our coordinate system and we set $e_{3}$ as the tangent direction $\tau$.

Let $v$ be a direction different from $\tau$. Consider a line $s(t)=v t$ approaching the origin as $t$ goes to zero. There is a positive number $t_{0}$ such that $s(t) \in \Omega \backslash\left(\bar{\sigma} \cup \overline{\sigma^{\prime}}\right)$ for $0<t<t_{0}$, hence

$$
u_{\sigma}(s(t))=w(s(t))+u_{\sigma^{\prime}}(s(t))=u_{\sigma^{\prime}}(s(t))
$$

is bounded for $t \in\left(0, t_{0}\right)$, since $d\left(s(t), \sigma^{\prime}\right)>0$. We want to show that this is a contradiction to the fact that $\nabla u_{0} \neq 0$.

The Neumann function $N$ can be written as

$$
N(x, y)=\Gamma(|x-y|)+h(x, y),
$$

where $\Gamma(|x-y|)=\frac{1}{4 \pi \gamma_{0}|x-y|}$ and $h$ is a harmonic function in $\Omega$. By inserting expression (36) into (34) we have that, for $t \in\left(0, t_{0}\right)$,

$$
\begin{aligned}
u_{\sigma}(s(t)) & =-\left(\gamma_{1}(s(t))-\gamma_{0}\right) \frac{\partial u_{0}}{\partial \tau}(s(t)) \Gamma(|s(t)|) \\
& -\int_{\sigma} \frac{\partial}{\partial \tau}\left(\left(\gamma_{1}-\gamma_{0}\right) \frac{\partial u_{0}}{\partial \tau}\right) \Gamma(|x-s(t)|) d \sigma_{x} \\
& +\int_{\sigma}\left(\gamma_{1}-\gamma_{0}\right) m(x) \widetilde{\nabla u_{0}}(x) \cdot \widetilde{\nabla \Gamma}(x, s(t)) d \sigma_{x}+\tilde{h}(t),
\end{aligned}
$$

where $\tilde{h}(t)$ is a bounded function.

Let $B_{0}=(0, R)$ be a ball centered at the origin with radius $R$, such that $0<R<|\sigma| / 2$ and $B_{0} \subset K_{0}$. Let us estimate the right term of (37).

Let us introduce by $v_{0}(x)=\left(\gamma_{1}(x)-\gamma_{0}\right) \partial u_{0}(x) / \partial \tau$. By the regularity assumptions on $u_{0} \in$ $C^{2}(\Omega)$ and $\gamma_{1}$, the function $v_{0}$ and its derivatives are bounded on $K_{0}$.

We first consider the first term in (37), which we rewrite in the following wat

$$
-\left(\gamma_{1}(s(t))-\gamma_{0}\right) \frac{\partial u_{0}}{\partial \tau}(s(t)) \Gamma(|s(t)|)=-v_{0}(0) \Gamma(|s(t)|)-\left(v_{0}(s(t))-v_{0}(0)\right) \Gamma(|s(t)|) .
$$


Note that the last right-hand-side term in $(38)$ is bounded for $t \in\left(0, t_{0}\right)$ due to the regularity of $v_{0}$.

We now write

$$
\begin{aligned}
& \int_{\sigma} \frac{\partial v_{0}}{\partial \tau}(x) \Gamma(|x-s(t)|) d \sigma_{x}=\int_{\sigma \backslash B_{0}} \frac{\partial v_{0}}{\partial \tau}(x) \Gamma(|x-s(t)|) d \sigma_{x} \\
& +\int_{\sigma \cap B_{0}}\left(\frac{\partial v_{0}}{\partial \tau}(x)-\frac{\partial v_{0}}{\partial \tau}(0)\right) \Gamma(|x-s(t)|) d \sigma_{x}+\frac{\partial v_{0}}{\partial \tau}(0) \int_{\sigma \cap B_{0}} \Gamma(|x-s(t)|) d \sigma_{x} \\
& :=I_{1}+I_{2}+I_{3}
\end{aligned}
$$

For $x \in \sigma \backslash B_{0}$ and $t<R / 2$, it is true that $|x-s(t)| \geq R-t \geq R / 2$ and therefore

$$
\left|I_{1}\right| \leq \frac{C}{R}
$$

On the other hand, because of the regularity of $v_{0}$, we can estimate

$$
\left|I_{2}\right| \leq C \int_{0}^{R} \frac{x_{3}}{\left[t^{2}\left(v_{1}^{2}+v_{2}^{2}\right)+\left(x_{3}-t v_{3}\right)^{2}\right]^{1 / 2}} d x_{3} \leq C \quad \text { for } \quad t \in\left[0, t_{0}\right]
$$

Last integral in (39) can be explicitly calculated and estimated by

$$
\left|I_{3}\right| \leq C\left|\ln \left(\frac{R}{t}\right)\right|
$$

Now, let us turn to the last term in (37). Arguing as before, we divide it into three parts, and this time we introduce by $V_{0}(x):=\left(\gamma_{1}-\gamma_{0}(x)\right) m(x) \widetilde{\nabla u_{0}}(x)$.

$$
\begin{aligned}
& \int_{\sigma} V_{0}(x) \cdot \widetilde{\nabla \Gamma}(x, s(t)) d \sigma_{x}=\int_{\sigma \backslash B_{0}} V_{0}(x) \cdot \widetilde{\nabla \Gamma}(x, s(t)) d \sigma_{x} \\
& +\int_{\sigma \cap B_{0}}\left(V_{0}(x)-V_{0}(0)\right) \cdot \widetilde{\nabla \Gamma}(x, s(t)) d \sigma_{x}+V_{0}(0) \cdot \int_{\sigma \cap B_{0}} \widetilde{\nabla \Gamma}(x, s(t)) d \sigma_{x} \\
& :=J_{1}+J_{2}+J_{3}
\end{aligned}
$$

For $x \in \sigma \backslash B_{0}$ and $t<R / 2$ we have that $|x-s(t)| \geq R-t \geq R / 2$ and, hence,

$$
\left|J_{1}\right| \leq \frac{C}{R^{2}}
$$

By regularity of $V_{0}$, we can estimate

$$
\left|J_{2}\right| \leq C \int_{0}^{R} \frac{x_{3} t}{\left[t^{2}\left(v_{1}^{2}+v_{2}^{2}\right)+\left(x_{3}-t v_{3}\right)^{2}\right]^{3 / 2}} d x_{3} \leq C \quad \text { for } \quad t \in\left[0, t_{0}\right] .
$$

Now, we evaluate term $J_{3}$ that contains a singularity of leading order:

$$
\begin{aligned}
J_{3} & =V_{0}(0) \cdot \int_{0}^{R} \frac{\left(-v_{1} t,-v_{2} t\right)}{\left[t^{2}\left(v_{1}^{2}+v_{2}^{2}\right)+\left(x_{3}-t v_{3}\right)^{2}\right]^{3 / 2}} d x_{3} \\
& =-V_{0}(0) \cdot \frac{\left(v_{1}, v_{2}\right)}{\left(v_{1}^{2}+v_{2}^{2}\right)}\left(\frac{z}{\sqrt{1+z^{2}}} \mid \frac{\frac{R-v_{3} t}{\sqrt{v_{1}^{2}+v_{2}^{2}} t}}{\frac{-v_{3}}{\sqrt{v_{1}^{2}+v_{2}^{2}}}}\right) \\
& \left.=-V_{0}(0) \cdot \frac{\left(v_{1}, v_{2}\right)}{\left(v_{1}^{2}+v_{2}^{2}\right) t} v_{3}+O\left(t \ln \left(\frac{1}{t}\right)\right)\right) .
\end{aligned}
$$

Collecting all this estimates we conclude that, for sufficiently small $t$,

$$
u_{\sigma}(s(t))=\left(\gamma_{1}(0)-\gamma_{0}\right)\left(-\frac{\partial u_{0}}{\partial \tau}(0) \frac{C_{1}}{t}+m(0) \widetilde{\nabla u_{0}}(0) \cdot \frac{\left(v_{1}, v_{2}\right)}{\sqrt{v_{1}^{2}+v_{2}^{2}} t} v_{3}\right)+O\left(\ln \frac{1}{t}\right) .
$$

The remainder term is the contr Since the function $u_{\sigma}(s(t))$ has to be bounded for $t \in\left(0, t_{0}\right)$ and for any direction $v \neq e_{3}$, we can choose $v_{3}=0$ in (40) and conclude that

$$
\frac{\partial u_{0}}{\partial \tau}(0)=\frac{\partial u_{0}}{\partial x_{3}}(0)=0
$$


Now let us choose a direction $v$ such that $v_{3} \neq 0$, and obtain

$$
m(0) \widetilde{\nabla u_{0}}(0) \cdot\left(v_{1}, v_{2}\right)=0 \quad \text { for any } \quad\left(v_{1}, v_{2}\right) \in \mathbb{R}^{2},
$$

which, in turn, implies that

$$
m(0) \widetilde{\nabla u_{0}}(0)=0 \text {. }
$$

Now, we notice that $\nabla u_{0}(0)=\left(\frac{\partial u_{0}}{\partial x_{1}}(0), \frac{\partial u_{0}}{\partial x_{2}}(0)\right)$ and that $m(0)$ is a symmetric and positive definite tensor, from which, together with (41), it follows that

$$
\nabla u_{0}(0)=0
$$

which contradicts our assumptions.

Let us now consider the case of a smooth coefficient $\gamma_{0}$. The Neumann function defined by (5) has the same singularities of function $\Gamma$ ( see $[15$, ch.1, sec.8]) and the same estimates can be carried out.

\section{Concluding REMARKS}

In this paper we proved that the polarization tensor of cylindrical inclusions can be deducted from the polarisation tensor of cross section orthogonal to the axis of the cylinder. When conductivity in the background and in the cylinder vary smoothly, the polarisation tensor in every cross section is only a function of the contrast $\gamma_{1} / \gamma_{0}$ in that cross section, and can be obtained by a 2-dimensional calculation. Note that our arguments do not depend on the dimension, and does not require the base to be of small diameter. For example, iterating this result between dimension 1 and dimension $d$, we would recover the polarisation tensor of a flat thin plate, already obtained in $[5,8]$, from that of a small segment in dimension 1.

The case of a base of small diameter is new, and we show that it allows uniquely determine the axis of the cylinder from one boundary measurement. We believe that a similar form of the polarization tensor holds for small neighborhoods of a general smooth curve. In this case the singularities of the correction term along the curve should be sufficient to be able to determine the curve itself from the knowledge of boundary data. This will be subject of a forthcoming paper.

\section{APPENDIX}

Lemma 5.1. [8, 2] Let $a \in L^{\infty}(\Omega)$ such that $c_{1}<a<c_{1}^{-1}$ for some positive constant $c_{1}$. Suppose that $\phi_{\epsilon} \in H^{1}(\Omega)$ is such that

$$
\operatorname{div}\left(a \nabla \phi_{\epsilon}\right)=\operatorname{div}\left(F_{\epsilon}\right) \text { in } \Omega
$$

where either

$$
\begin{aligned}
F_{\epsilon} & =1_{\omega_{\epsilon}}(x) F_{0}(x) \text { with }\left\|F_{0}\right\|_{L^{\infty}\left(\omega_{\epsilon}\right)^{d}} \leq F_{C} \\
\text { or } F_{\epsilon} & =1_{\omega_{\epsilon}}(x) F_{\epsilon}(x) \text { with }\left\|F_{\epsilon}\right\|_{L^{2}(\Omega)^{d}} \leq F_{C}\left|\omega_{\epsilon}\right|^{1 / 2} .
\end{aligned}
$$

where $F_{C}$ is a constant independent of $\epsilon$. Then,

$$
\left\|\nabla \phi_{\epsilon}\right\|_{L^{2}(\Omega)^{d}} \leq \frac{1}{\sqrt{c_{0}}}\left|\omega_{\epsilon}\right|^{1 / 2} F_{C},
$$

Furthermore, there exists $\alpha>0$ and $C>0$, independent on $\epsilon$, such that

$$
\left\|\phi_{\epsilon}\right\|_{L^{2}(\Omega)} \leq C\left|\omega_{\epsilon}\right|^{\frac{1}{2}+\alpha} F_{C} .
$$




\section{REFERENCES}

[1] H. Ammari, E. Beretta, E. Francini, Reconstruction of thin conductivity imperfections, Applicable Analysis, 83(1), 63-76, (2004).

[2] H. Ammari, E. Bonnetier, Y. Capdeboscq, M. Tanter and M. Fink, Electrical Impedance Tomography by Elastic Deformation, SIAM J. Appl. Math., 68(6), 1557-1573, 2008.

[3] H. Ammari and H. Kang, Reconstruction of Small Inhomogeneities from Boundary Measurements, volume 1846 of Lecture Notes in Mathematics, Springer, 2004.

[4] —, Polarization and Moment Tensors with Applications to Inverse Problems and Effective Medium Theory, Applied Mathematical Sciences Series 162, Springer, Berlin, 2007.

[5] E. Beretta, E. Francini, and Michael S. Vogelius, Asymptotic formulas for steady state voltage potentials in the presence of thin inhomogeneities. A rigorous error analysis, J. Math. Pures Appl. (9), 82(10),1277-1301, 2003.

[6] E. Beretta, A. Mukherjee, and M. S. Vogelius, Asymptotic formulas for steady state voltage potentials in the presence of conductivity imperfections of small area, Z. Angew. Math. Phys., 52, 543-572, 2001.

[7] Y. Capdeboscq and M. S. Vogelius, A general representation formula for boundary voltage perturbations caused by internal conductivity inhomogeneities of low volume fraction. M2AN Math. Model. Numer. Anal., 37(1), 159-173, 2003.

[8] Y. Capdeboscq and M. S. Vogelius, Pointwise polarization tensor bounds, and applications to voltage perturbations caused by thin inhomogeneities, Asymptotic Analysis, 50, 175-204, 2006.

[9] D. J. Cedio-Fengya, S. Moskow, and M. S. Vogelius, Identification of conductivity imperfections of small diameter by boundary measurements. continuous dependence and computational reconstruction, Inverse Problems, 14, 553-595, 1998.

[10] A. Friedman and M. Vogelius, Identification of small inhomogeneities of extreme conductivity by boundary measurements: a theorem on continuous dependence, Arch. Rat. Mech. Anal. 105 (1989), 299-326.

[11] D. Gilbarg and N. S. Trudinger, Elliptic Partial Differential Equations of Second Order, Comprehensive Studies in Mathematics. Springer-Verlag, 2nd edition, 1983.

[12] V. Kozlov, V. Maz'ya, and A. Movchan, Asymptotic analysis of fields in multi-structures, Oxford Mathematical Monographs. Oxford University Press, 1999.

[13] R. Lipton, Inequalities for electric and elastic polarization tensors with applications to random composites, $J$. Mech. Phys. Solids, 41(5), 809-833, 1993.

[14] G. W. Milton, The Theory of Composites, Cambridge Monographs on Applied and Computational Mathematics, Cambridge University Press, 2002.

[15] C. Miranda, Partial Differential Equations of Elliptic type, Ergebnisse der Mathematik und ihrer Grenzgebiete, Band 2. Springer-Verlag, New York-Berlin, 1970.

[16] A. B. Movchan and S. K. Serkov, The Pólya-Szegő matrices in asymptotic models of dilute composites. European J. Appl. Math., 8(6) (1997), 595-621.

[17] G. Pólya and G. Szegö. Isoperimetric Inequalities in Mathematical Physics, Annals of Mathematics Studies, no. 27, Princeton University Press, Princeton, N. J., 1951.

[18] M. Schiffer and G. Szegö, Virtual mass and polarization. Trans. Amer. Math. Soc., 67 (1949), 130-205.

DIPARTIMENTO DI MATEMATICA "G. CASTELNUOVO" UNIVERSITÀ DI ROMA "LA SAPIENZA", PIAZZALE ALDO MORO 5, 00185 ROMA, ITALY

E-mail address: BERETTA@MAT. UNIROMA1.IT

MATHEMATICAL INSTITUTE, UNIVERSITY OF OXFORD, OXFORD OX1 3LB, UK

E-mail address: CAPDEBOSCQ@MATHS.OX.AC.UK

DiPARTIMENTO DI MATEMATICA "U. DINI", VIALE MORGAGNI 67A, 50134 FIRENZE, ITALY

E-mail address: FRANCINI@MATH.UNIFI.IT 